INPLASY

PROTOCOL

To cite: Tasso et al.

Association between Candida

Albicans and Squamous Cell

Carcinoma: A Systematic

Literature Review. Inplasy

protocol 202130005. doi:

10.37766/inplasy2021.3.0005

Received: 02 March 2021

Published: 02 March 2021

Corresponding author:

Túlio Morandin Ferrise

tuliomferrisse@gmail.com

Author Affiliation:

São Paulo State University

(Unesp), School of Dentistry,

Araraquara

Support: Capes.

Review Stage at time of this submission: Preliminary

searches.

Conflicts of interest:

None declared.

\section{Association between Candida Albicans and Squamous Cell Carcinoma: A Systematic Literature Review}

Tasso, CO1; de Oliveira, AB2; Ferrisse, TM³; Jorge, $\mathrm{JH}^{4}$.

Review question / Objective: P - Patients with lesions of oral squamous cell carcinoma E - candida albicans oral infection C - Patients with lesion of oral squamous cell carcinoma without oral infection by candida albicans $O$ - The presence of oral infection by candida albicans is a factor in the progression of lesions of oral squamous cell carcinoma.

Condition being studied: The relationship between the Candida albicans microorganism and squamous cell carcinoma will be studied. Whether there is an influence on the initiation / progression of the disease when Candida albicans is associated.

Information sources: PUBMED; SCOPUS; EMBASE; WEB OF SCIENCE and SCIENTIFIC DIRECT.

INPLASY registration number: This protocol was registered with the International Platform of Registered Systematic Review and Meta-Analysis Protocols (INPLASY) on 02 March 2021 and was last updated on 02 March 2021 (registration number INPLASY202130005).

\section{INTRODUCTION}

Review question / Objective: P - Patients with lesions of oral squamous cell carcinoma E - candida albicans oral infection C - Patients with lesion of oral squamous cell carcinoma without oral infection by candida albicans 0 - The presence of oral infection by candida albicans is a factor in the progression of lesions of oral squamous cell carcinoma.

Rationale: The existence of a causality between the presence of Candida albicans in the initiation / evolution of squamous cell carcinoma is not elucidated. 
Condition being studied: The relationship between the Candida albicans microorganism and squamous cell carcinoma will be studied. Whether there is an influence on the initiation / progression of the disease when Candida albicans is associated.

\section{METHODS}

Search strategy: ((Oral cancer OR oral squamous cell carcinoma) AND (candida albicans)) AND (Carcinogen OR carcinogenesis) Databases: PUBMED; SCOPUS; EMBASE; WEB OF SCIENCE and SCIENTIFIC DIRECT.

Participant or population: Patients with squamous cell carcinoma.

Intervention: Candida albicans oral infection.

Comparator: Patients with lesion of oral squamous cell carcinoma without oral infection by candida albicans.

Study designs to be included: Clinical studies, animal studies and in vitro studies.

Eligibility criteria: The inclusion criteria for this systematic review were: studies in animal models, clinical trials and in vitro studies, studies that evaluated the effect of the presence of oral infection by candida albicans on the progression of oral squamous cell carcinoma and articles written in English. In addition, the exclusion criteria were: other experimental study designs such as review articles, letters to the editor, personal opinions, book chapters or conference abstracts, studies that did not have a control group, articles written in other languages and articles whose full text is not available for free.

Information sources: PUBMED; SCOPUS; EMBASE; WEB OF SCIENCE and SCIENTIFIC DIRECT.

Main outcome(s): As effect measurements, we going to perform the mean difference.This review will make an important contribution as oral cancer is the sixth most common type of cancer in the world, affecting more than 300,000 people annually, with an increasing incidence and high mortality rate $(62 \%)$. The relationship between oral cancer and prosthetic stomatitis should be evaluated, since understanding these relationships could help prevent both diseases. In addition, the effect that treatments directed at cancer patients have on the oral microbiota should be investigated, enabling the prevention of supporting diseases, such as prosthetic stomatitis, which, in debilitated patients, can cause systemic diseases. Different types of cancer treatment can also lead to changes in the oral cavity due to local and / or systemic factors (Lalla et al. 2010). These changes include immunosuppression (drug-induced or disease), imbalance in the oral microbiota (for example, secondary to antibiotic therapy), hyposalivation (drug-induced, disease or local radiation) and local tissue damage (for example, mucositis secondary to chemotherapy and / or radiation therapy). Cancer patients receiving chemotherapy and / or radiation therapy are more prone to all of the aforementioned predisposing factors and are therefore considered a risk group for oral fungal infections (Lalla et al. 2010;)

Data management: Mendeley - Reference Management software e Researcher.

Quality assessment / Risk of bias analysis: For analysis of the quality of clinical trial articles, the Delphi list will be used and for bias analysis, the Rob 2 scale will be used. For bias analysis of animal models, the SYRCLE scale will be used.

Strategy of data synthesis: A table with data extraction from included articles will be perform with the following itens: author and year, study design, sample size, control group .After that, with there was possibility to form groups, the metaanalyzes will be conducted using the random effect model.

Subgroup analysis: The possibility of perform sub-group analyzes will be evaluated after the data extraction. 
Sensitivity analysis: The sensitivity analyzes will be perform if the meta-analyzes report high heterogeneity (12>50\%). For that, the Baujat's plot will be made.

Language: Only articles in the English language will be included.

Country(ies) involved: Brazil.

Keywords: Oral squamous cell carcinoma; candida albicans.

Dissemination plans: One paper will be made from the results found in the systematic review.

Contributions of each author:

Author 1 - Camilla Olga Tasso.

Email: camillaotasso@gmail.com

Author 2 - Analú Barros de Oliveira.

Email: analuboliveira2@gmail.com

Author 3 - Túlio Morandin Ferrisse.

Email: tuliomferrisse@gmail.com

Author 4 - Janaina Habib Jorge.

Email: habib.jorge@unesp.br 